\title{
Influence of the Hall effect on the reconnection rate at line-tied magnetic X-points
}

\author{
I. J. D. Craig ${ }^{1}$ and Y. E. Litvinenko ${ }^{2}$ \\ 1 Department of Mathematics, University of Waikato, PB 3105, Hamilton, New Zealand \\ 2 Institute for the Study of Earth, Oceans, and Space, University of New Hampshire, Durham, 03824-3525, USA \\ e-mail: yuri.litvinenko@unh.edu
}

Received 13 February 2008 / Accepted 28 March 2008

\section{ABSTRACT}

\begin{abstract}
Context. The role of the Hall term in magnetic reconnection at line-tied planar magnetic $X$-points is explored.
Aims. The goal is to determine the reconnection scaling laws and to investigate how the reconnection rate depends on the size of the system in Hall magnetohydrodynamics (MHD).

Methods. The evolution of reconnective disturbances is determined numerically by solving the linearized compressible Hall MHD equations. Scaling laws are derived for the decay rate as a function of the dimensionless resistivity and ion inertial length.

Results. Although the Hall effect leads to an increase in the decay rate, this increase is shown to be moderated in larger systems. A key finding is that the Hall term contribution to the decay rate, normalized by the resistive decay rate, scales inversely with the system size $L$, approximately as $L^{-2}$.

Conclusions. The evidence suggests that decay rate enhancements due to Hall effects in line-tied $X$-points are weakened for largescale systems. The result may have important implications for modeling energy release in large-scale astrophysical plasma environments, such as solar flares.
\end{abstract}

Key words. magnetohydrodynamics (MHD) - Sun: flares - Sun: magnetic fields

\section{Introduction}

An area of active research in space physics is the inclusion of collisionless effects in resistive magnetohydrodynamic (MHD) models for magnetic reconnection (e.g., Shay et al. 2001; Craig et al. 2003; Morales et al. 2005; Daughton et al. 2006; Karimabadi et al. 2007). Such studies are motivated by the fact that resistive reconnection time scales are too slow to explain the observed reconnection times, for example, in solar flares (e.g., Cassak et al. 2006) or the solar wind (e.g., Gosling et al. 2005; Dasso et al. 2007).

Numerical simulations suggest that the Hall term in the generalized Ohm's law may play a key role in speeding up the reconnection rate, essentially independent of other model assumptions and the codes employed (Birn et al. 2001; Birn et al. 2005). In particular, Hall MHD may provide the simplest physical model for describing fast magnetic reconnection in weakly collisional plasmas. The plasma resistivity is still required to extract energy from the plasma and alter the magnetic field topology but the Hall term acts indirectly by controlling the advection of the magnetic field into the reconnection region.

Recently it has been argued that the Hall term can speed up reconnection rates in a manner that is independent of the size of the reconnecting system (Shay et al. 1999; Cassak et al. 2006). This claim, if correct, would have important implications for solar flare physics. Suppose, for instance, that $\alpha_{0}$ is the rate of energy decay in the absence of Hall effects in a system of global scale $L$. Then if $\Delta \alpha>0$ is the change in the rate when the Hall term is included, we would have

$\frac{\Delta \alpha}{\alpha_{0}} \sim L^{p}$

with $p=0$ if the claim were correct. On the other hand, $p<0$ would compromise the speed-up for large-scale systems such as a powerful solar flare. This is why the system-size dependence remains a hotly debated topic in the literature.

It is interesting that analytical Hall MHD models for steady incompressible reconnection (Dorelli 2003; Craig \& Watson 2005) can be used to estimate $p=-1$, suggesting that Hall effects become less effective for larger scales. This result cannot be regarded as definitive, however, because steady analytical models indicate that the Hall term may either speed up or slow down the rate, depending on the orientation of an imposed axial guide field. Also computations performed using the conventional reconnection symmetries show that a key signature of Hall merging is the generation of transient axial field components of quadrupolar structure. Such structures are not adequately represented in the simplified steady analytical models.

In this paper we explore another possibility for determining the system-size dependence, based on incorporating Hall effects within a transient $X$-point collapse model (Senanayake \& Craig 2006). This approach models the energy decay of a closed compressible line-tied $X$-point and has the advantage of complementing the more customary incompressible periodic geometry (Berger 1997; Watson \& Craig 2001). Our aim is twofold: first, 
to extend the $X$-point computations initiated by Senanayake \& Craig (2006) to obtain more detailed reconnection scalings; second, to use these scalings to explore the system-size dependence of the reconnection rate in Hall MHD.

\section{Hall MHD reconnection at a planar magnetic $X$-point}

We work with the compressible Hall MHD equations, scaled with respect to typical values of the magnetic field strength $B_{0}$, the global length scale (system size) $L$, and the number density $n_{0}$. Times are then measured in units of the global Alfvén time $L / v_{\mathrm{A}}$, where $v_{\mathrm{A}}=B_{0} /\left(4 \pi m_{\mathrm{p}} n_{0}\right)^{1 / 2}$ is the characteristic Alfvén speed. For example, $B_{0}=10^{2} \mathrm{G}, L=10^{9.5} \mathrm{~cm}, n_{0}=10^{9} \mathrm{~cm}^{-3}$, and $v_{\mathrm{A}}=10^{9} \mathrm{~cm} \mathrm{~s}^{-1}$ can be adopted for a solar active region. ity

Resistive effects are controlled by the dimensionless resistiv-

$\eta=\frac{c^{2}}{4 \pi v_{\mathrm{A}} L \sigma} \simeq 10^{-14.5}$,

based on the collisional conductivity $\sigma \sim T^{3 / 2}$. The role of the Hall effect is quantified by the dimensionless ion inertial length

$d_{\mathrm{i}}=\frac{c}{L \omega_{\mathrm{pi}}} \simeq 10^{-6.5}$.

Here $\omega_{\mathrm{pi}}=\left(4 \pi n_{0} e^{2} / m_{\mathrm{p}}\right)^{1 / 2}$ is the proton plasma frequency.

We consider small displacements of a cold (pressureless) plasma in a $2 \frac{1}{2}$-dimensional problem with $\partial_{z}=0$. We use the flux function representation for $\boldsymbol{B}(x, y, t)$, and we split the magnetic and velocity fields into planar and axial components:

$\boldsymbol{B}(x, y, t)=\left(\partial_{y} \psi,-\partial_{x} \psi, Z\right), \quad \boldsymbol{v}(x, y, t)=(\boldsymbol{u}, W)$

where $\boldsymbol{u} \cdot \hat{z}=0$.

The evolution equations are obtained by linearizing about an equilibrium solution $\boldsymbol{B}=\boldsymbol{B}_{\mathrm{E}}$ and $\boldsymbol{v}=0$. The equilibrium state is a planar potential $X$-point of uniform density, specifically

$\psi_{\mathrm{E}}=x y, \quad-1 \leq x, y \leq 1$

with $v=0, Z=0$. As far as the initial planar magnetic field is concerned, we let

$\psi(x, y, t) \rightarrow \psi_{\mathrm{E}}(x, y)+\psi(x, y, t)$

and perturb the planar field at $t=0$ according to

$\psi(x, y, 0)=0.1\left(1-x^{2}\right)\left(1-y^{2}\right)$.

This disturbance raises the magnetic energy of the $X$-point and drives reconnection by changing the magnetic topology. The boundary conditions are specified by field line-tying to rigid walls where $\boldsymbol{v}=0$ and $\psi=0$. The derivative of $Z$ normal to the boundary is also assumed to vanish.

The resulting first-order system of dimensionless Hall MHD equations provides a model for describing $X$-point Hall magnetic reconnection:

$$
\begin{aligned}
& \frac{\partial \boldsymbol{u}}{\partial t}=-\nabla^{2} \psi \nabla \psi_{\mathrm{E}}+v \boldsymbol{F}_{\perp}, \\
& \frac{\partial W}{\partial t}=\left(\boldsymbol{B}_{\mathrm{E}} \cdot \nabla\right) Z+v F_{\|}, \\
& \frac{\partial \psi}{\partial t}+\boldsymbol{u} \cdot \nabla \psi_{\mathrm{E}}=\eta \nabla^{2} \psi-d_{\mathrm{i}}\left(\boldsymbol{B}_{\mathrm{E}} \cdot \nabla\right) Z,
\end{aligned}
$$

$$
\frac{\partial Z}{\partial t}=\left(\boldsymbol{B}_{\mathrm{E}} \cdot \nabla\right) W+\eta \nabla^{2} Z+d_{\mathrm{i}}\left(\boldsymbol{B}_{\mathrm{E}} \cdot \nabla\right) \nabla^{2} \psi .
$$

Note that, in contrast to Senanayake \& Craig (2006), we allow for the possibility that a classical viscous force $\boldsymbol{F}=\left(\boldsymbol{F}_{\perp}, F_{\|}\right)$ may act on the velocity field (Craig et al. 2005).

The presence of the Hall term $\left(d_{\mathrm{i}}>0\right)$ means that it is generally impossible to consider a purely planar reconnection problem with $Z=W=0$. According to Eq. (11), gradients in the axial electric current $\nabla^{2} \psi$ along the equilibrium magnetic field $\boldsymbol{B}_{\mathrm{E}}$ will induce growth in the axial magnetic field $Z$, which in turn will generate an axial velocity $W$ according to Eq. (9). The electric current density $\boldsymbol{J}=\left(Z_{y},-Z_{x},-\nabla^{2} \psi\right)$ will develop planar components in addition to the axial reconnection-related component of conventional MHD. These effects will influence both the reconnection rate, defined as $\partial_{t} \psi=\eta\left(\nabla^{2} \psi\right)_{0}$ at the $X$-point, and the Ohmic dissipation rate $\eta \int J^{2} \mathrm{~d} V$.

The phenomenology of the resulting transient magnetic reconnection - including the generation of induced quadrupolar axial fields - has already been discussed (Senanayake \& Craig 2006) and will not be pursued here. Our present focus is to determine the reconnection scaling laws and to investigate how the decay rate in the presence of the Hall term is affected by the size of the system.

\section{Hall MHD reconnection scalings}

First recall the purely resistive case in the absence of Hall and viscous effects $\left(d_{\mathrm{i}}=0\right)$. The reconnection problem can be understood in terms of an analytical $X$-point solution (Craig \& McClymont 1991, 1993; Hassam 1992). The key result is that the resistive decay of the solution is dominated by the fundamental cylindrical eigenmode $\psi \sim \exp (\mathrm{i} \lambda t)$ with an eigenvalue

$$
\lambda=i \alpha_{0}+\omega_{0}, \quad \alpha_{0}=\frac{1}{2} \omega_{0}^{2}, \quad \omega_{0}=\frac{\pi}{|\ln \eta|}
$$

for sufficiently small $\eta$. The weak (logarithmic) dependence of the eigenvalue on the resistivity shows that the solution provides a fast reconnection model, characterized by a rapid decay $\sim \exp \left(-\alpha_{0} t\right)$ even for very small resistivities. Physically, the fundamental oscillation frequency $\omega_{0}$ follows from the time it takes for a cylindrical Alfvén wave, launched from the outer boundary $(r \simeq 1)$, to reach a diffusion region of scale $r_{\mathrm{s}} \simeq \eta^{1 / 2}$. The analytical result (12) provides only an approximate scaling for the present problem because the fundamental cylindrical mode requires a circular, as opposed to a square outer boundary. Our reconnection simulations in the case $d_{\mathrm{i}}=0$ show that the oscillation frequency and the decay rate conform very well to the analytical prediction. Moreover, including classical viscosity with $v<\eta$ does not compromise these findings (Craig et al. 2005).

The conditions under which the Hall term becomes important can be assessed by comparing $\eta \nabla^{2} \psi$ with $d_{\mathrm{i}}\left(\boldsymbol{B}_{\mathrm{E}} \cdot \nabla\right) Z$ in Eq. (10). Using Eq. (11) to estimate $Z$, we find that Hall effects should come into play when $\tau d_{\mathrm{i}}^{2} / \eta \geq 1$, where $\tau$ is a typical dynamical timescale. This condition should be easily met in the solar corona where $d_{\mathrm{i}}^{2} / \eta \simeq 10^{1.5} \gg 1$, even for very fast Alfvénic relaxation with $\tau=O(1)$. In our computations, $\tau \simeq \pi / \omega_{0} \simeq 10$. Hence Hall term effects should already dominate when $d_{\mathrm{i}} / \eta^{1 / 2} \simeq 1$

Physically, $d_{\mathrm{i}}$ gives the thickness of a current sheet, determined by collisionless effects, whereas $\eta^{1 / 2}$ gives the thickness of the classical Sweet-Parker current sheet or a more general flux pile-up current sheet (e.g., Litvinenko \& Craig 2003). In the problem at hand, $\eta^{1 / 2}$ determines the radius at which resistive 


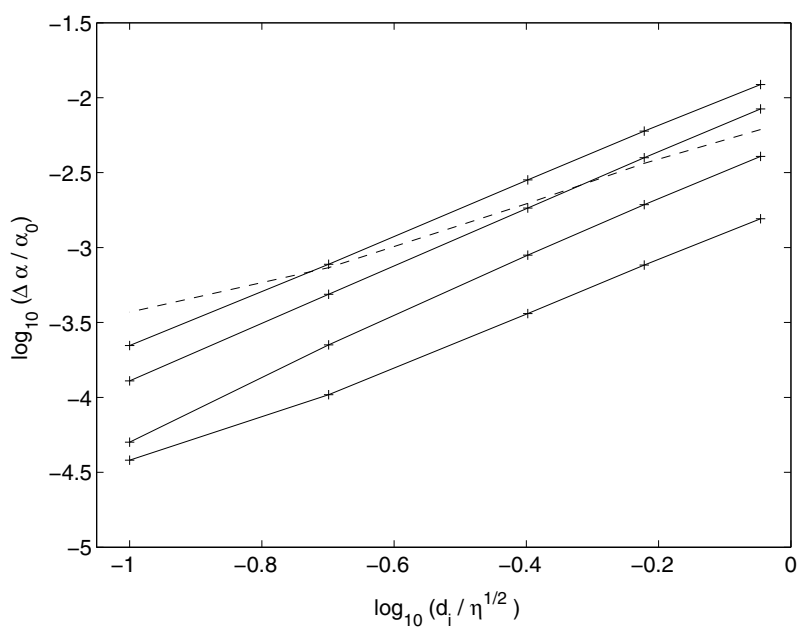

Fig. 1. Plot of $\log \left(\Delta \alpha / \alpha_{0}\right)$ against $\log \kappa$ where $\kappa=d_{\mathrm{i}} / \sqrt{\eta}$ for runs with $1.5 \times 10^{-4} \leq \eta \leq 2 \times 10^{-2}$. Data for $\eta \geq 10^{-2}$ are typified by the dashed line $\left(\eta=2 \times 10^{-2}\right)$.

diffusion becomes important. Since the measure $\Delta \alpha / \alpha_{0}$ defines the relative roles of the Hall and resistive effects in the energy decay, it might be expected that this ratio be some function of the Hall length scale $d_{\mathrm{i}}$ and the resistive length scale $\eta^{1 / 2}$. The actual dependencies are explored below using a least squares method to fit the computational data.

Turning now to the problem of computing $\Delta \alpha / \alpha_{0}$, we note that the original results of Senanayake \& Craig (2006) are not well-suited to this task because they cover a wide range of Hall coefficients over a modest range of resistivities. In such cases the scaling of the current layer is not known a priori and it is difficult to maintain consistent numerical resolution. The strategy we adopt here uses an extended range of resistivies but restricts the computation to modest $d_{\mathrm{i}} / \eta^{1 / 2} \leq 1$, so that the scale $r_{\mathrm{s}} \simeq \eta^{1 / 2}$ remains influential. Despite this restriction, induced axial fields can be a substantial fraction of the reconnecting planar components. Data sets constructed in this way have the advantage of allowing consistent resolution over a wide parameter range, possibly at the cost of introducing cancellation errors in $\Delta \alpha$ for very small $d_{\mathrm{i}}$. The data are then fitted by the method of least squares, using a function of the form

$\log \left(\Delta \alpha / \alpha_{0}\right)=c_{1}+c_{2} \log d_{\mathrm{i}}+c_{3} \log \eta$

All data sets are based on the same initial conditions given by Eq. (7).

Figure 1 summarizes the raw data of over twenty runs covering the range $1.5 \times 10^{-4} \leq \eta \leq 2 \times 10^{-2}$ with $d_{\mathrm{i}}=\kappa \sqrt{\eta}$, where $0.1 \leq \kappa \leq 0.9$ and $v=0.1 \eta$. Note that runs for small $\eta$ are computationally expensive because of the well known time step limitations introduced by Hall effects even for modest $d_{\mathrm{i}}$. At the other end of the range, data for large resistivities are in danger of lying outside of an asymptotic regime. Thus, as illustrated by the dotted line in Fig. 1, data for $\eta \geq 10^{-2}$ contradict the systematic trend found at lower resistivities. Accordingly, these data are discarded in the final least squares fit that gives

$\log \left(\Delta \alpha / \alpha_{0}\right)=-0.16+1.89 \log d_{\mathrm{i}}-0.31 \log \eta$.

This result, illustrated in Fig. 2, implies a strong $d_{\mathrm{i}}$ dependence, yet is remarkably insensitive to the resistivity.

Our results suggest a scaling law of the form

$\frac{\Delta \alpha}{\alpha_{0}} \simeq A\left(\frac{d_{\mathrm{i}}}{\eta^{1 / 6}}\right)^{a}$

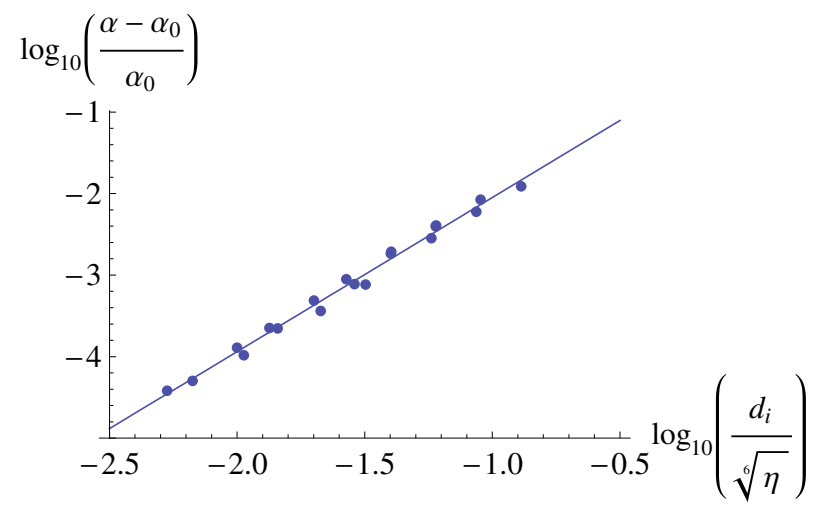

Fig. 2. Plot of $\log \left(\Delta \alpha / \alpha_{0}\right)$ against $\log \left(d_{\mathrm{i}} / \eta^{1 / 6}\right)$ for runs with $1.5 \times 10^{-4} \leq$ $\eta \leq 10^{-2}$ and $0.1 \leq \kappa \leq 0.9$. The linear least-squares fit is $y=-0.16+$ $1.89 x$.

with $A \simeq 0.7$ and $a \simeq 2$. Moreover, subsidiary computations strongly suggest that the overall tendency is a weakening of the $\eta$ dependence as further data at lower resistivity levels are introduced.

\section{System-size dependence of the reconnection rate}

As noted in the introduction, the dependence of the magnetic reconnection rate (typically nondimensionalized by the global Alfvén time) on the system size is a matter of strong practical interest. One point of view, suggested by numerical models with periodic boundary conditions and analytical scaling arguments, is that the length of the current sheet is independent of the system size when the Hall term controls the reconnection physics (Shay et al. 1999; Cassak et al. 2006). Mass continuity then dictates that the Hall reconnection rate must be a universal constant that is independent of the system size. If this claim were correct, it would effectively solve the longstanding problem of finding a reconnection mechanism that is fast enough to explain the explosive energy release in solar flares. This claim, however, has recently been questioned (Bhattacharjee et al. 2005). In addition, recent kinetic simulations of reconnection with open boundary conditions show that the length of the electron diffusion region increases with time, leading to the formation of an extended current sheet (Daughton et al. 2006). This is in sharp contrast to numerical Hall MHD models with periodic boundary conditions.

Motivated by the consideration that different reconnection geometries may be a factor that influences the reconnection rate, we now use the present results to explore the system-size dependence. This is easily achieved because the dimensionless parameters $\eta$ and $d_{\mathrm{i}}$ depend on $L$ according to Eqs. (2) and (3). Note that any function of $d_{\mathrm{i}} / \eta$ is independent of $L$. However, using Eq. (15) and neglecting the very weak $\eta$ dependence, we find that

$\frac{\Delta \alpha}{\alpha_{0}} \sim d_{\mathrm{i}}^{2} \sim L^{-2}$

where we have set $a=2$ for simplicity. Hence $p=-a=-2$ in Eq. (1). The key point is that the reconnection time $\sim \alpha^{-1}$ at linetied X-points, as a multiple of the Alfvén time, will be longer for larger systems. 


\section{Discussion}

In this paper we explored the effect of an increase of the magnetic reconnection rate in Hall MHD, relative to the wellestablished resistive reconnection scalings. Specifically, we solved linearized compressible Hall MHD equations that describe reconnection at a line-tied magnetic $X$-point. While periodic boundary conditions are typically employed in numerical studies of collisionless reconnection, our results are obtained in a topologically unambiguous $X$-point geometry.

Our main result concerns the dependence of the reconnection rate on the system size (global length scale). The Hall term contribution to the dimensionless decay rate $\Delta \alpha$ is found to scale as $L^{-2}$ with the system size $L$. Remarkably, this scaling derives from a quadratic dependence on the ion inertial length $d_{\mathrm{i}}$ yet is almost independent of the dimensionless resistivity. This reconnection scaling implies, for instance, that smaller loops in the solar corona can sustain stronger reconnection electric fields $E \simeq \alpha v_{\mathrm{A}} B_{0} / c$.

Our results are qualitatively consistent with an exact selfsimilar solution for the current sheet formation in Hall MHD, which predicts the sheet formation time that is an increasing function of a global length scale (Litvinenko 2007). They are also broadly consistent with exact steady incompressible Hall MHD solutions which suggest that Hall effects can both speed up and slow down magnetic reconnection, depending on the structure of the magnetic field (Dorelli 2003; Craig \& Watson 2005). It should be emphasized that numerical (e.g., Morales et al. 2005) and analytical (e.g., Craig et al. 2003) time-dependent studies of Hall MHD reconnection often invoke plasma incompressibility. Our approach, based on compressible Hall MHD, should therefore provide a more realistic description of reconnection in a strongly compressible magnetized plasma of the solar corona.

To summarize, our analysis does not support the viewpoint that the Hall effect controls the reconnection physics in such a way that the ratio of the reconnection time and a global Alfvén time is a universal constant independent of the system size (cf., Shay et al. 1999; Cassak et al. 2006). Independent kinetic simulations of collisionless reconnection with open boundary conditions also show the formation of an extended current sheet, implying a system-size dependence of the reconnection rate (Daughton et al. 2006; Karimabadi et al. 2007). While more work is needed to resolve the controversy, these results suggest that a rich variety of collisionless reconnection regimes could exist, depending on the magnetic geometry involved and the choice of initial and boundary conditions.

Acknowledgements. This work was supported by NSF (grant ATM-0734032), NASA (grants NNG05GM43G, NNX07AI04G, and NNX08AG44G), the Marsden Fund (02-UOW-050 MIS), and a research fellowship from the Alexander von Humboldt Foundation. Comments by the anonymous referee are gratefully acknowledged.

\section{References}

Berger, M. A. 1997, J. Geophys. Res., 102, 2637

Bhattacharjee, A., Germaschewski, K., \& Ng, C. S. 2005, Phys. Plasmas, 12, 042305

Birn, J., Drake, J. F., Shay, M. A., et al. 2001, J. Geophys. Res., 106, 3715

Birn, J., Galsgaard, K., Hesse, M., et al. 2005, Geophys. Res. Lett., 32, L06105 Biskamp, D., \& Welter, H. 1980, Phys. Rev. Lett., 44, 1069

Cassak, P. A., Drake, J. F., \& Shay, M. A. 2006, ApJ, 644, L145

Craig, I. J. D., \& McClymont, A. N. 1991, ApJ, 371, L41

Craig, I. J. D., \& McClymont, A. N. 1993, ApJ, 405, 207

Craig, I. J. D., \& Watson, P. G. 2005, Phys. Plasmas, 12, 12306

Craig, I. J. D., Heerikhuisen, J., \& Watson, P. G. 2003, Phys. Plasmas, 10, 3120

Craig, I. J. D., Litvinenko, Y. E., \& Senanayake, T. 2005, A\&A, 433, 1139

Daughton, W., Scudder, J., \& Karimabadi, H. 2006, Phys. Plasmas, 13, 072101

Dasso, S., Nakwacki, M. S., Démoulin, P., \& Mandrini, C. H. 2007, Solar Phys., 244,115

Dorelli, J. C. 2003, Phys. Plasmas, 10, 3309

Gosling, J. T., Skoug, R. M., McComas, D. J., \& Smith, C. W. 2005, J. Geophys. Res., 110, A01107

Hassam, A. B. 1992, ApJ, 399, 159

Karimabadi, H., Daughton, W., \& Scudder, J. 2007, Geophys. Res. Lett., 34, 13104

Knoll, D. A., \& Chacon, L. 2006, Phys. Rev. Lett., 96, 135001

Litvinenko, Y. E. 2007, Phys. Plasmas, 14, 112303

Litvinenko, Y. E., \& Craig, I. J. D. 2003, Solar Phys., 218, 173

Morales, L. F., Dasso, S., \& Gómez, D. O. 2005, J. Geophys. Res., 110, A04204

Rickard, G. J., \& Craig, I. J. D. 1993, Phys. Fluids B, 5, 956

Shay, M. A., Drake, J. F., Rogers, B. N., \& Denton, R. E. 1999, Geophys. Res. Lett., 26, 2163

Shay, M. A., Drake, J. F., Rogers, B. N., \& Denton, R. E. 2001, J. Geophys. Res., 106,3759

Senanayake, T., \& Craig, I. J. D. 2006, A\&A, 451, 1117

Watson, P. G., \& Craig, I. J. D. 2001, J. Geophys. Res., 106, 15735 